\title{
Genotype by environment interactions in identifying cassava (Manihot esculenta Crantz) resistant
}

\section{to cassava brown streak disease}

Emily A. Masinde a,b, Geoffrey Mkamillo ${ }^{\mathrm{c}}$, Joshua O. Ogendo ${ }^{\mathrm{a}}$, Rory Hillocks ${ }^{\mathrm{b}}$, Richard M.S. Mulwa ${ }^{\mathrm{a}}$, Bernadetta Kimata ${ }^{\mathrm{c}}$, Midatharahally N. Maruthi $\mathbf{b}^{*}$

a'Department of Crops, Horticulture and Soils, Egerton University, P.O. Box 536-20115, Egerton, Kenya.

${ }^{b}$ Natural Resources Institute, University of Greenwich, Chatham Maritime, Kent ME4 4TB, UK.

'Naliendele Agricultural Research Institute (NARI), P.O Box 509, Mtwara, Tanzania.

Key words: Cassava; Landraces; CBSD resistance; Genotype x environment interaction; Yield related traits

*Corresponding author

Email address: m.n.maruthi@gre.ac.uk

Postal address: Natural Resources Institute, University of Greenwich, Chatham Maritime, Kent ME4 4TB, UK.

Email addresses: Emily A. Masinde (masinde.emily11@gmail.com), Joshua O. Ogendo (ogendojoshua@gmail.com), Rory Hillocks (roryhillocks@yahoo.co.uk), Richard M.S. Mulwa (isamuk@gmail.com), Bernadetta Kimata (bkimatha@ yahoo.co.uk), Geoffrey Mkamillo (geoffreymkamilo@yahoo.co.uk), Midatharahally N. Maruthi (m.n.maruthi@gre.ac.uk) 


\section{ABSTRACT}

Cassava landraces were evaluated for resistance to cassava brown streak disease (CBSD) for two cropping seasons at a disease hotspot area in Naliendele, Tanzania. Based on reactions to CBSD, several landraces including Chimaje, Mfaransa and Supa B were considered to be resistant to the disease while Kikwada, Mbuyu, and Nyoka were tolerant. ANOVA revealed that the largest sum of squares (SS) (41.9 - 86.7\%) was attributed to the genotype of the cassava landraces, while a smaller proportion of SS (8.1 - 38.2\%) was due to genotype by environment interactions for all traits tested, which included disease symptoms, root weight, number of roots per plant and dry matter content. Environment accounted for the smallest effect $(0.01-26.3 \%)$, however, the mean squares was nonetheless significant for a few genotypes, which indicated that their disease expression was indeed influenced by the environment. Increased CBSD severity was associated with low temperatures and rainfall. Increased rainfall towards harvesting led to higher root weight but lower dry matter content in the first cropping season. Correlation analysis showed that the presence of CBSD symptoms reduces the amount of usable roots, total root weight, and root dry matter content. Many resistant/tolerant landraces also had high root weight and dry matter content, and they can be used by farmers to reduce CBSD losses. The landraces described here form novel sources of CBSD resistance that can be used for breeding disease-resistant cassava varieties with superior agronomic characteristics. 


\section{Introduction}

Cassava brown streak disease (CBSD) is arguably the most dangerous threat to cassava (Manihot esculenta Crantz), which is Africa's most important food security crop. The disease causes losses to cassava root production and quality. Cassava brown streak virus (CBSV) (Monger et al., 2001) and Ugandan cassava brown streak virus (UCBSV) belonging to the genus Ipomovirus, famly Potyviridae cause CBSD (Mbazibwa et al., 2009). Both viruses, together called cassava brown streak ipomoviruses (CBSIs), have a positive-sense single stranded RNA genome (Winter et al., 2010; Ndunguru et al., 2015). CBSD symptoms include foliar chlorosis and necrosis, brown streaks on stems, constrictions and dry corcky necrotic rot of roots and stunted plant growth (Hillocks and Jennings, 2003; Winter et al., 2010; Vanderschuren et al., 2012). CBSIs are mainly spread by the propagation of infected cassava cuttings by farmers, but also transmitted in a semi-persistent manner by the insect vector whiteflies, Bemicia tabaci (Gennadius) (Maruthi et al., 2005, 2017; Mware et al., 2009).

Early reports on CBSD distribution identified the disease to be mostly restricted to the East African coast and the shores of Lake Malawi (Nichols, 1950). For many years, it was believed that the disease does not spread at altitudes 1000m above sea level (Nichols, 1950; Hillocks et al., 1999). However, in recent years, outbreaks of CBSD have been reported at mid altitude levels (1200 - 1500 meters above sea levels) in Uganda, western Kenya and Tanzania, Mozambique, Rwanda, Burundi, and in isolated parts of the Democratic Republic of Congo (Mahungu et al., 2003; Alicai et al., 2007; Jeremiah and Legg, 2008; Osogo et al., 2014). CBSD is currently estimated to cause annual economic losses in excess of US\$ 726 million (Maruthi M N, unpublished data). A definitive cause for the sudden upsurge in CBSD incidences is yet to be identified, however, the introduction of the virus to mid-altitude areas and the presence of high whitefly populations are considered to be the key drivers of new CBSD outbreaks (Legg et al., 2011, 2014).

CBSD symptom expression and resistance to the virus depends on the type of the variety (resistant or susceptible), growing conditions (temperature, rainfall, altitude etc.), age of the plant and the virus isolate involved in causing the symptoms (Mohammed et al., 2012). The 
genetic factor of the plant is by far the biggest contributor to symptom determination and disease severity (Nichols, 1950). Cassava breeding for disease resistance began at the East African Cassava Research Institute at Amani in northern Tanzania (Nichols, 1950; Hillocks and Jennings, 2003). The search for resistance led to breeders introgressing disease resistance through interspecific crosses with wild cassava Manihot spp. (Jennings, 1957). Crosses with Manihot glaziovii backcrossed three times and intercrossed with resistant hybrids produced interspecific hybrids that were rated as highly resistant to another major cassava disease called cassava mosaic disease (CMD) but moderately resistant to CBSD (Jennings, 1957). Some of the best-known intercrosses at Amani included cultivars 46106/27, 5318/34 and 5543/156 (Jennings, 1994). Hybrid 46106/27, also known as Amani in Tanzania showed high levels of field resistance to CBSD. 46106/27 is closely related to, but not identical to, a Tanzanian local cultivar called Namikonga (Kulembeka et al., 2012; Pariyo et al., 2013). Namikonga was, therefore, considered to be an interspecific hybrid from the Amani program that was subsequently adopted by the farming communities and given a local name. Namikonga has been consistently resistant to CBSD for many years and has the highest general combining ability for disease resistance (Nichols, 1947; Kulembeka et al., 2012; Masumba et al., 2017). Some of the present day so-called "local cultivars" in Tanzania, especially the few which have proved to be resistant/tolerant to CBSD, including Kigoma Red, Kigoma Mafia and Kiroba are also likely to have some pedigree related to the Amani breeding programme (Mahungu et al., 1998; Kanju et al., 2010; Pariyo et al., 2015; Bredeson et. al., 2016; Masumba et. al., 2017; Nzuki et. al., 2017). The best CBSD resistance genotypes are likely to have survived in farmer fields as landraces in Tanzania from the Amani research programme. Research has also shown that some of these CBSD-resistant genotypes have performed well in multiple locations, adapting to different agro-ecologies and disease pressures (Abaca et al., 2012; Pariyo et al., 2015). For example, Kigoma Red a local landrace is resistant to CBSD in both Tanzania and Uganda. Identifying and saving such germplasm is therefore important for controlling the disease in the affected African countries.

At present, Namikonga still expresses field resistance to CBSD and is used as one of the best sources of CBSD resistance in conventional breeding programs (Kanju et al., 2010; Pariyo et al. 2013; Maruthi et al. 2014; Masumba et al., 2017). Bredeson et al. (2016) reported 
Namikonga, TMS130572, KBH2006/18, Mkombozi, TMS 1972205 and Akena to have a common M. glaziovii haplotype on chromosome 1 designated as the 'Amani haplotype'. For a long time Kiroba was perceived as an Amani hybrid but Nzuki et al. (2017) reported that its $M$. glaziovii haplotype is different from that of Namikonga and it has a close parent-offspring relationship with tree cassava. When the Amani program ceased in around 1958, it is thought that some of the inter specific crosses found their way into farmer's fields in Tanzania and have been incorporated as farmer varieties (Kanju et al., 2003). The clones may have lost their identities and are being grown by farmers under different local names. Although cassava breeders have identified some interspecific hybrids that show strong levels of resistance/tolerance to CBSD, there are still many unidentified clones which could potentially be good sources of CBSD resistance/tolerance. This work was aimed to address this gap with the intention of identifying best CBSD resistant cassava landraces particularly focusing on clones that do not develop root necrosis (Legg et al., 2011). New and diverse sources of resistance are required to combat the newly emerged threat by CBSD in which mixed infections of both CBSV and UCBSV are common both in mid and low altitude areas of eastern and southern African regions (Adams et al., 2013; Osogo et al., 2014; Ndunguru et al., 2015).

\section{Materials and methods}

\subsection{Cassava germplasm and screening location}

Sixty-four local landraces were collected from farmer's fields in 12 administrative districts in the Southern and Eastern zones of Tanzania (Tandahimba, Mtwara rural, Newala, Masasi, Lindi urban, Lindi rural, Kilwa, Mtwara Rural, Mtwara, Urban, Rufiji and Mkuranga). These were established and multiplied at the Naliendele Agricultural Research Institute (NARI), Tanzania for CBSD resistance screening in open fields. Cassava varieties Kiroba (CBSD tolerant) and Albert (CBSD susceptible) available at NARI germplasm collections were used as controls.

In the literature, many terminologies have been used inconsistently to describe the response of cassava to CBSD, and in general of plants to virus infections (Cooper and Jones, 1983). Among them, resistance or field resistance, tolerance or field tolerance, and susceptibility are most common. Resistance or field resistance commonly indicates lack of virus multiplication, no or mild symptoms and no reduction in yield. 
Tolerant varieties can support virus multiplication, exhibit mild to severe symptoms but no significant reduction in yield, while the susceptible varieties support high levels of virus multiplication, exhibit severe symptoms and suffer significant yield loss (Hillocks and Jennings, 2003; Maruthi et al., 2014). For simplicity, the terminologies resistance and tolerance were used in this study also to represent field resistance and field tolerance, respectively.

Screening of germplasm for CBSD infection in the field was conducted in 2014 and 2015 cropping seasons at NARI, which is a hot spot for CBSD infection. Naliendele lies on the coastal belt of the Indian Ocean and is located at $10^{\circ} 22^{\prime} 20^{\prime \prime} \mathrm{S}, 40^{\circ} 10^{\prime} 34^{\prime \prime E}$ and $111 \mathrm{~m}$ above sea level. The area receives rainfall from December-May with scattered showers in August-October (TMA, 2009). The soils are characterised by very deep, well drained, weak structured, dark reddish brown loamy sand topsoil over a reddish brown moderately structured sandy loam to sandy clay loam subsoil (Mugogo and Njapuka, 2007).

\subsection{Experimental design}

Partially balanced lattice with three replicates was used for this study. Fifteen cassava cuttings (about $25 \mathrm{~cm}$ long and having 4 to 5 nodes with viable buds) from each of the local landraces and the controls (Kiroba and Albert) were planted at spacing of $1.0 \mathrm{~m} \times 1.0 \mathrm{~m}$ in January 2014 . To increase CBSD inoculum pressure, cuttings from a known susceptible and infected genotype Albert were planted after every 10 rows of the test genotypes to act as a disease spreader. In addition, the first and last rows were also planted with infected Albert cuttings to prevent border effects. This configuration ensured that every plant is exposed to similar high inoculum pressure and no plant escapes infection. No fertiliser or irrigation was provided (typical rain-fed crop) but kept weed-free throughout the growing period. The trial was repeated in 2015 cropping season. 


\subsection{CBSD symptoms on leaves and roots}

CBSD leaf symptoms incidence and severity was recorded at 3, 6, and 9 months after planting (MAP). The disease severity was scored on 1 - 5 scale (Fig.1) (Hillocks et al., 1996). Plants germinated with disease symptoms were removed to allow uniform symptom development across all the varieties. At $12 \mathrm{MAP}$, plants from each landrace were harvested (excluding the border plants) and roots examined for symptoms. Roots from each landrace were chopped longitudinally and transversely to identify the presence of necrotic patches on the starch bearing tissues. Scoring for root necrosis severity was also done based on a 1 - 5 scale (Fig. 2) (Gondwe et al., 2002). Data on root necrosis incidence was collected with incidences recorded from a root necrosis score of $\geq 2$. Since CBSD mostly affects root quality, usable roots (palatable and marketable) per genotype was determined by cutting out the necrotic tissues and weighing the unaffected roots. All roots with necrosis score of $\leq 2$ were considered to be fully usable as only tiny spots of root necrosis were observed at this score. This is also the general practice followed by Tanzanian farmers. The weight of usable roots was expressed as percentage of the total root weight. Data were collected on quantitative traits such as root weight in tonnes per hectare, number of roots per plant and dry matter content at 12 MAP.

Root yield in tonnes per hectare (t/ha/) was estimated according to Kamau et al., (2011).

Root yield $\left(\frac{t}{h a}\right)=\frac{\left[\text { root weight }\left(\frac{\mathrm{kg}}{\mathrm{m} 2}\right) \times 10000\right]}{1000}$

Root dry matter content was calculated using the specific gravity method (Kawano, 1987).

Dry matter content $(\%)=158.3 \times\left[\frac{\text { weight in air }}{\text { weight in air }- \text { weight in } \text { water }}\right]-142$ 

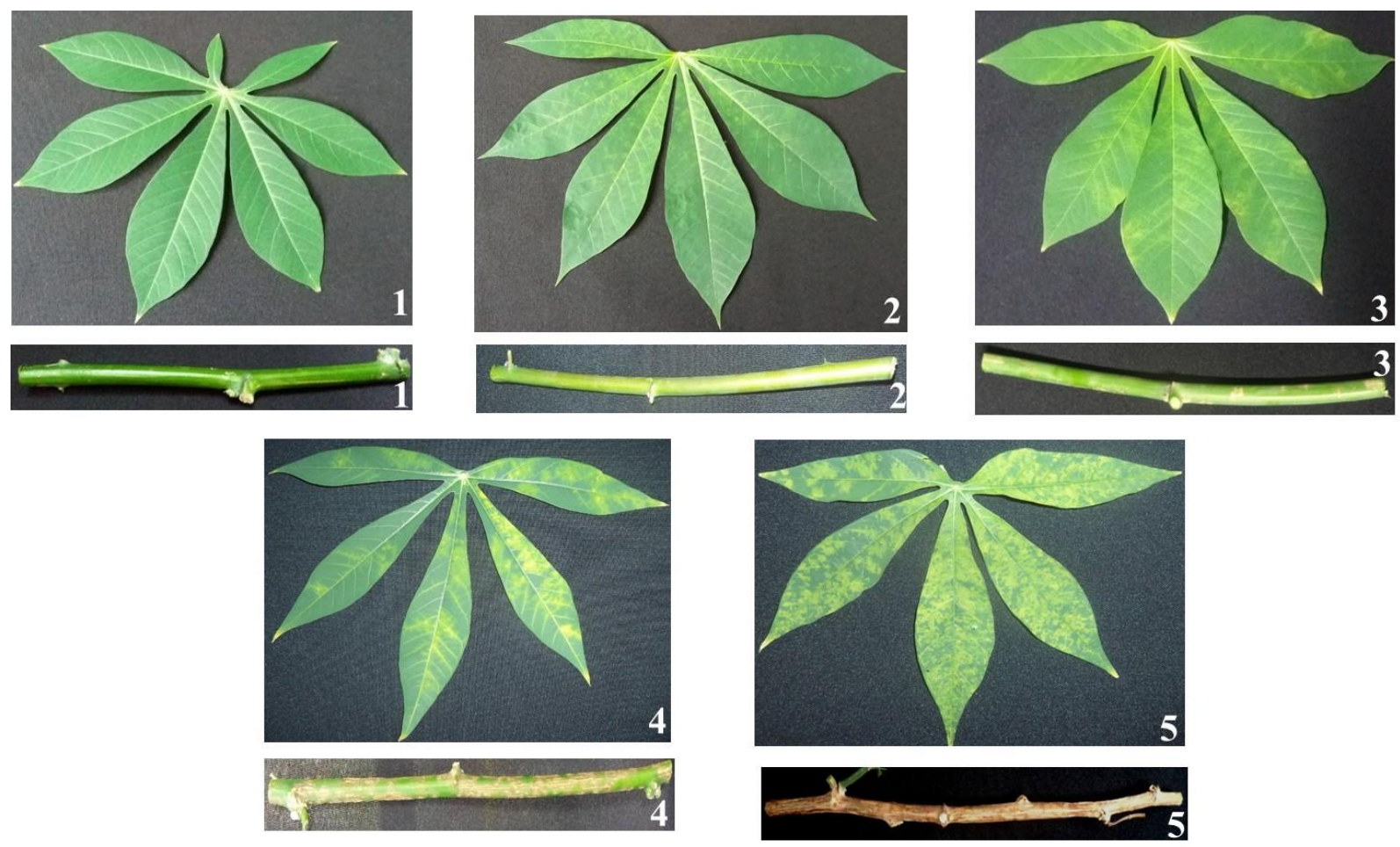

Fig. 1. CBSD symptom severity scale $1-5$ used for recording sympotms on cassava leaves. $1=$ no apparent symptoms, $2=$ slight foliar mosaic, no stem lesions, 3 = foliar mosaic, mild stem lesions no die back, $4=$ foliar mosaic and pronounced stem lesions no die back, and $5=$ defoliation with stem lesions and pronounced die back. 

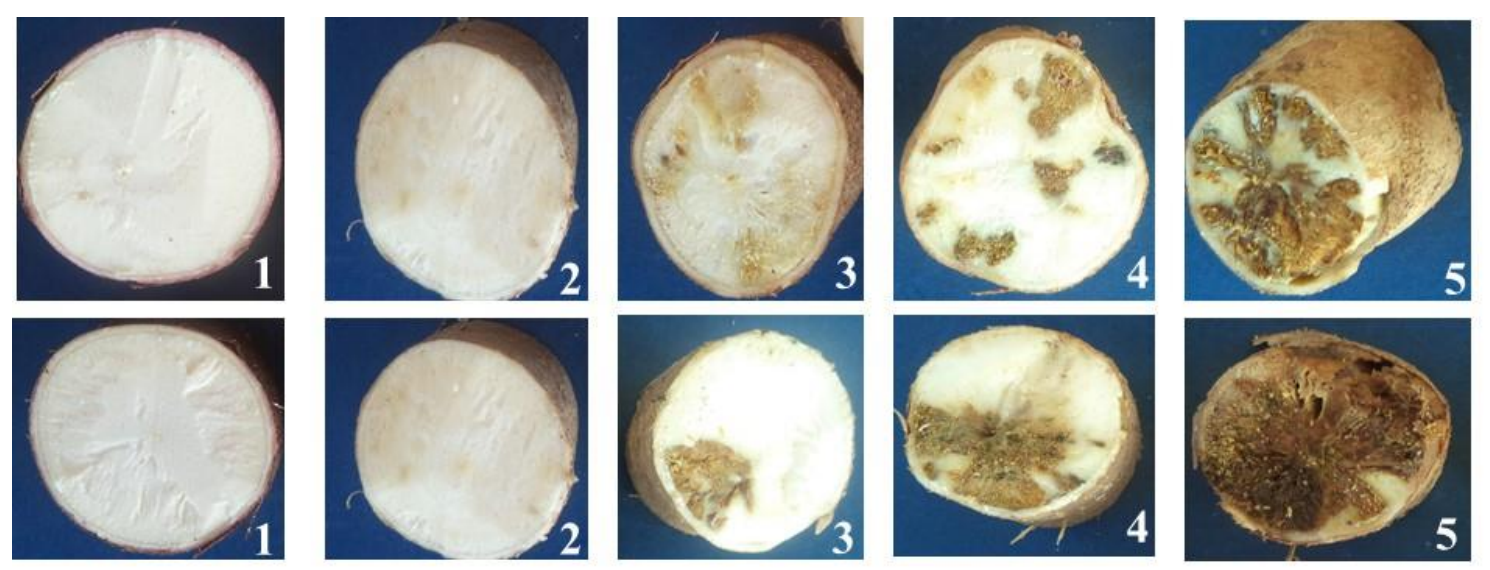

Fig. 2. CBSD root necrosis symptoms severity scale $1-5$ used for recording root symptoms. $1=$ no apparent symptoms, $2=<5 \%$ of root necrotic, $3=5-25 \%$ of root necrotic, $4=25-50 \%$ root necrotic and mild root constriction, and $5=>50 \%$ of root necrotic with severe root constriction. Roots with only scores of $\leq 2$ were considered marketable yields.

\subsection{Data analysis}

The landraces were classified into three categories based on foliar and root necrosis severity scores;

$1.0-2.0$ were considered resistant,

$2.1-3.0$ were considered tolerant, and

$3.1-5.0$ were considered susceptible.

The landraces were also classified on the basis of root necrosis incidence;

$0-10 \%$ were categorized as resistant,

$11-40 \%$ were categorized as tolerant, and

$41-100 \%$ were categorized as susceptible. 
These two sets of data were used together for classifying the varieties as described previously (Hillocks and Jennings, 2003). Resistant plants showed low foliar symptoms while tolerant plants readily expressed foliar symptoms but with low root necrosis severity (Hillocks et al., 2001). Susceptible varieties showed severe foliar and root symptoms with high levels of disease incidences.

Analysis of variance was carried out on means of three replications for CBSD leaf symptoms severity at 3, 6 and 9 MAP, root necrosis severity, root necrosis incidence, usable roots (\%), root weight in tonnes per hectare, dry matter content, and harvest index. Combined ANOVA was carried out for the two cropping seasons and treatment means separated using Dunnett's method to compare each local landrace against control (Kiroba), at 95\% confidence level. Spearman's correlation analysis was carried out to determine the correlation between CBSD symptoms, root weight, dry matter content, and harvest index. All analysis was carried out using the Statistical Analysis System (SAS) (SAS Institute Inc, 2013).

\section{Results}

\subsection{CBSD foliar symptoms}

CBSD mean foliar severity increased throughout the growing seasons. The mean leaf severity was 1.4 (on a scale of 1 to 5) at 3 MAP, 1.8 at 6 MAP, and 1.9 at 9 MAP (Table 1) across all the varieties tested. At 3 MAP, most landraces had low mean foliar severity ranging from 1.0 to 1.9 , which was not significantly $(\mathrm{P} \leq 0.05)$ different from the tolerant control Kiroba, whose mean foliar severity was 1.3 . However, Kigoma Red, Kitumbua, Mbuyu, Nachinyaya, Ntonto, and Nyankagile had significantly $(\mathrm{P} \leq 0.05)$ higher mean foliar severity ranging from 2.4 to 2.8 . At 6 MAP, Kiroba had a mean foliar severity of 2.1, and while several landraces had significantly lower mean foliar severity ranging from 1.2 to 1.5 (Table 1). In contrast, landraces Kigoma Red, Kikombe, Kitumbua, Mbuyu, Ntonto, and Nyankagile had higher mean foliar severity ranging from 2.7 to 3.1. At 9 MAP, Kiroba had a mean foliar severity of 2.1, while Benny, Chimaje, Katewanya, Likonde, Limbanga, Liumbukwa, and Mnacho had significantly lower mean foliar severity ranging from 1.1 to 1.3. Both tolerant (Kiroba) and susceptible (Albert) controls had mean foliar severity that were not significantly different from each other at 3, 6, and 9 MAP (Table 1). 


\subsection{CBSD root symptoms}

The mean root necrosis severity was 1.4 (on a scale of 1 to 5) across all the landraces evaluated. Kiroba had a low mean root necrosis severity of 1.0 and it was not significantly $(\mathrm{P} \leq 0.05)$ different from landraces including Azoa, Benny, Chimaje, Chipanda, Kikwada, Likonde, Mombasa, Nyoka, and Supa B (Table 1). Albert together with other landraces including Bangi, Cosmas, Hingawali, Kalinda, Kigoma Red, and Sumu ya Panya had significantly higher mean root necrosis severity ranging from 1.4 to 3.4.

Table 1

Means of CBSD foliar symptoms at 3,6 and 9 MAP, root symptoms and other root traits.

\begin{tabular}{|c|c|c|c|c|c|c|c|c|c|}
\hline Landraces & $\begin{array}{l}\text { Foliar } \\
\text { symptoms } \\
\text { at } 3 \text { MAP }\end{array}$ & $\begin{array}{l}\text { Foliar } \\
\text { symptoms at } \\
6 \mathrm{MAP}\end{array}$ & $\begin{array}{l}\text { Foliar } \\
\text { symptoms } \\
\text { at } 9 \text { MAP }\end{array}$ & $\begin{array}{l}\text { Root } \\
\text { necrosis }\end{array}$ & $\begin{array}{l}\text { Root necrosis } \\
\text { incidence }(\%)\end{array}$ & $\begin{array}{l}\text { Usable } \\
\text { roots } \\
(\%)\end{array}$ & $\begin{array}{l}\text { Root weight } \\
(\mathrm{t} / \mathrm{ha})\end{array}$ & $\begin{array}{l}\text { Root } \\
\text { number/ } \\
\text { plant }\end{array}$ & $\begin{array}{l}\text { Dry matter } \\
\text { content } \\
(\%)\end{array}$ \\
\hline Kiroba & 1.3 & 2.1 & 2.2 & 1.0 & 0.0 & 100.0 & 22.4 & 5.4 & 27.2 \\
\hline Albert & $1.3^{\mathrm{ns}}$ & $1.9^{\mathrm{ns}}$ & $1.8^{\mathrm{ns}}$ & $3.4^{* * * *}$ & $99.2^{* * * *}$ & $53.2^{* * * *}$ & $12.7^{* * * *}$ & $4.3^{\mathrm{ns}}$ & $24.2^{\mathrm{ns}}$ \\
\hline Azoa & $1.1^{\mathrm{ns}}$ & $1.2^{* * * *}$ & $1.8^{\mathrm{ns}}$ & $1.1^{\mathrm{ns}}$ & $3.8^{\mathrm{ns}}$ & $98.3^{\mathrm{ns}}$ & $15.8^{*}$ & $3.6^{*}$ & $29.2^{\mathrm{ns}}$ \\
\hline Bangi & $1.4^{\mathrm{ns}}$ & $1.7^{\mathrm{ns}}$ & $1.4^{*}$ & $1.4^{*}$ & $5.5^{\mathrm{ns}}$ & $92.2^{\mathrm{ns}}$ & $21.4^{\mathrm{ns}}$ & $5.8^{\mathrm{ns}}$ & $28.5^{\mathrm{ns}}$ \\
\hline Benny & $1.1^{\mathrm{ns}}$ & $1.3^{* * *}$ & $1.2^{* * *}$ & $1.2^{\mathrm{ns}}$ & $2.3^{\mathrm{ns}}$ & $99.0^{\mathrm{ns}}$ & $19.5^{\mathrm{ns}}$ & $5.1^{\mathrm{ns}}$ & $33.2^{* * *}$ \\
\hline Binamuli & $1.3^{\mathrm{ns}}$ & $1.7^{\mathrm{ns}}$ & $1.8^{\mathrm{ns}}$ & $1.1^{\mathrm{ns}}$ & $7.6^{* * *}$ & $98.7^{\mathrm{ns}}$ & $13.3^{* * * *}$ & $5.6^{\mathrm{ns}}$ & $37.3^{* * *}$ \\
\hline Binti Ally & $1.1^{\mathrm{ns}}$ & $1.7^{\mathrm{ns}}$ & $2.0^{\mathrm{ns}}$ & $1.4^{*}$ & $14.3^{* * * *}$ & $94.9^{\mathrm{ns}}$ & $18.6^{\mathrm{ns}}$ & $5.2^{\mathrm{ns}}$ & $26.5^{\mathrm{ns}}$ \\
\hline Binti Juma & $1.4^{\mathrm{ns}}$ & $2.0^{\mathrm{ns}}$ & $2.0^{\mathrm{ns}}$ & $1.1^{\mathrm{ns}}$ & $8.5^{* * *}$ & $89.5^{\text {** }}$ & $10.0^{* * * *}$ & $3.2^{* * * *}$ & $29.0^{\mathrm{ns}}$ \\
\hline Binti Pindi & $1.3^{\mathrm{ns}}$ & $1.5^{*}$ & $1.8^{\mathrm{ns}}$ & $1.0^{\mathrm{ns}}$ & $1.4^{\mathrm{ns}}$ & $98.5^{\mathrm{ns}}$ & $14.7^{* * * *}$ & $4.1^{\mathrm{ns}}$ & $29.8^{\mathrm{ns}}$ \\
\hline Chidubwa & $1.8^{\mathrm{ns}}$ & $2.4^{\mathrm{ns}}$ & $2.2^{\mathrm{ns}}$ & $1.2^{\mathrm{ns}}$ & $2.3^{\mathrm{ns}}$ & $99.0^{\text {ns }}$ & $15.4^{* * *}$ & $4.6^{\mathrm{ns}}$ & $32.2^{*}$ \\
\hline Chimaje & $1.3^{\mathrm{ns}}$ & $1.3^{* * *}$ & $1.1^{\text {*** }}$ & $1.0^{\mathrm{ns}}$ & $0.0^{\mathrm{ns}}$ & $100.0^{\mathrm{ns}}$ & $13.6^{* * * *}$ & $3.8^{\mathrm{ns}}$ & $30.4^{\mathrm{ns}}$ \\
\hline Chipanda & $1.3^{\mathrm{ns}}$ & $1.3^{\text {*** }}$ & $1.7^{\mathrm{ns}}$ & $1.0^{\mathrm{ns}}$ & $0.0^{\mathrm{ns}}$ & $100.0^{\mathrm{ns}}$ & $17.1^{\mathrm{ns}}$ & $5.4^{\mathrm{ns}}$ & $33.2^{* *}$ \\
\hline Cosmas & $1.8^{\mathrm{ns}}$ & $2.1^{\mathrm{ns}}$ & $2.4^{\mathrm{ns}}$ & $2.1^{* * *}$ & $54.3^{* * * *}$ & $69.9^{* * *}$ & $9.9^{* * *}$ & $3.9^{\mathrm{ns}}$ & $27.0^{\mathrm{ns}}$ \\
\hline Hamad Rashid & $1.1^{\mathrm{ns}}$ & $1.3^{* * *}$ & $1.6^{\mathrm{ns}}$ & $1.1^{\mathrm{ns}}$ & $3.8^{\mathrm{ns}}$ & $97.3^{\mathrm{ns}}$ & $4.7^{* * * *}$ & $3.5^{* *}$ & $27.3^{\text {ns }}$ \\
\hline Hingawali & $1.1^{\mathrm{ns}}$ & $1.7^{\mathrm{ns}}$ & $1.8^{\mathrm{ns}}$ & $1.6^{* * *}$ & $20.4^{* * * *}$ & $84.2^{* * * *}$ & $14.0^{* * * *}$ & $4.8^{\mathrm{ns}}$ & $26.8^{\mathrm{ns}}$ \\
\hline
\end{tabular}




\begin{tabular}{|c|c|c|c|c|c|c|c|c|c|}
\hline Kalinda & $1.5^{\mathrm{ns}}$ & $2.3^{\mathrm{ns}}$ & $2.2^{\mathrm{ns}}$ & $1.8^{* * *}$ & $26.6^{* * *}$ & $82.3^{* * * *}$ & $12.1^{* * *}$ & $4.5^{\mathrm{ns}}$ & $31.8^{*}$ \\
\hline Katewanya & $1.4^{\mathrm{ns}}$ & $1.5^{*}$ & $1.2^{* * * *}$ & $1.1^{\mathrm{ns}}$ & $3.5^{\mathrm{ns}}$ & $98.3^{\text {ns }}$ & $23.5^{\mathrm{ns}}$ & $4.3^{\mathrm{ns}}$ & $35.3^{* *}$ \\
\hline Kibangameno & $1.0^{\mathrm{ns}}$ & $1.9^{\mathrm{ns}}$ & $2.3^{\mathrm{ns}}$ & $1.5^{* * *}$ & $14.7^{* * * *}$ & $90.0^{*}$ & $13.2^{* * * *}$ & $2.9^{* * *}$ & $26.7^{\mathrm{ns}}$ \\
\hline Kifuu cha nazi & $1.0^{\mathrm{ns}}$ & $1.9^{\mathrm{ns}}$ & $2.1^{\mathrm{ns}}$ & $1.2^{\mathrm{ns}}$ & $11.7^{* * * *}$ & $99.7^{\mathrm{ns}}$ & $11.9^{* * *}$ & $3.9^{\mathrm{ns}}$ & $26.5^{\mathrm{ns}}$ \\
\hline Kigoma Red & $2.7^{* * *}$ & $3.1^{* * *}$ & $2.7^{\mathrm{ns}}$ & $3.4^{* * *}$ & $89.8^{* * * *}$ & $37.6^{* * *}$ & $16.1^{*}$ & $5.4^{\mathrm{ns}}$ & $28.2^{\mathrm{ns}}$ \\
\hline Kikombe & $1.7^{\mathrm{ns}}$ & $2.7^{* *}$ & $2.9^{\mathrm{ns}}$ & $2.0^{* * * *}$ & $45.0^{* * *}$ & $67.9^{* * *}$ & $9.6^{* * *}$ & $3.5^{* *}$ & $32.7^{\text {** }}$ \\
\hline Kikwada & $1.9^{\mathrm{ns}}$ & $2.2^{\mathrm{ns}}$ & $2.3^{\mathrm{ns}}$ & $1.0^{\mathrm{ns}}$ & $0.0^{\mathrm{ns}}$ & $100.0^{\mathrm{ns}}$ & $11.3^{* * *}$ & $4.0^{\mathrm{ns}}$ & $29.0^{\mathrm{ns}}$ \\
\hline Kitumbua & $2.4^{* * * *}$ & $2.8^{* * * *}$ & $2.9^{*}$ & $1.7^{* * * *}$ & $20.8^{* * * *}$ & $87.7^{* * * *}$ & $12.2^{* * *}$ & $4.4^{\mathrm{ns}}$ & $32.9^{\text {** }}$ \\
\hline Kiwinda & $1.5^{\mathrm{ns}}$ & $1.8^{\mathrm{ns}}$ & $2.0^{\mathrm{ns}}$ & $1.0^{\mathrm{ns}}$ & $0.0^{\mathrm{ns}}$ & $100.0^{\mathrm{ns}}$ & $13.3^{* * * *}$ & $3.4^{* * *}$ & $32.5^{* *}$ \\
\hline Likonde & $1.1^{\mathrm{ns}}$ & $1.0^{* * *}$ & $1.1^{* * *}$ & $1.2^{\mathrm{ns}}$ & $11.3^{* * *}$ & $94.0^{\mathrm{ns}}$ & $11.0^{* * * *}$ & $4.6^{\mathrm{ns}}$ & $31.0^{\mathrm{ns}}$ \\
\hline Likonde II & $1.7^{\mathrm{ns}}$ & $2.0^{\mathrm{ns}}$ & $2.2^{\mathrm{ns}}$ & $2.0^{* * * *}$ & $24.7^{* * *}$ & $81.8^{* * * *}$ & $21.7^{\mathrm{ns}}$ & $6.2^{\mathrm{ns}}$ & $25.2^{\mathrm{ns}}$ \\
\hline Limbanga & $1.1^{\mathrm{ns}}$ & $1.1^{* * * *}$ & $1.2^{* * * *}$ & $1.0^{\mathrm{ns}}$ & $0.0^{\text {ns }}$ & $100.0^{\mathrm{ns}}$ & $23.0^{\mathrm{ns}}$ & $5.6^{\mathrm{ns}}$ & $33.4^{* *}$ \\
\hline Liumbukwa & $1.0^{\mathrm{ns}}$ & $1.0^{* * * *}$ & $1.2^{* * *}$ & $1.9^{* * * *}$ & $19.5^{* * *}$ & $83.9^{* * * *}$ & $17.0^{\mathrm{ns}}$ & $5.3^{\text {ns }}$ & $36.4^{* * *}$ \\
\hline Makame & $1.0^{\mathrm{ns}}$ & $1.6^{\mathrm{ns}}$ & $1.9^{\mathrm{ns}}$ & $1.1^{\mathrm{ns}}$ & $2.3^{\text {ns }}$ & $92.8^{\mathrm{ns}}$ & $15.5^{* *}$ & $4.4^{\mathrm{ns}}$ & $29.7^{\mathrm{ns}}$ \\
\hline Mbuyu & $2.8^{* * *}$ & $3.0^{* * *}$ & $2.9^{\mathrm{ns}}$ & $1.0^{\mathrm{ns}}$ & $2.2^{\mathrm{ns}}$ & $100.0^{\mathrm{ns}}$ & $15.7^{*}$ & $4.0^{\mathrm{ns}}$ & $27.7^{\mathrm{ns}}$ \\
\hline Mdimbe & $1.4^{\mathrm{ns}}$ & $2.4^{\mathrm{ns}}$ & $1.9^{\mathrm{ns}}$ & $2.1^{* * * *}$ & $35.2^{* * *}$ & $69.7^{* * * *}$ & $20.7^{\mathrm{ns}}$ & $5.6^{\mathrm{ns}}$ & $28.5^{\mathrm{ns}}$ \\
\hline Mfaransa & $1.5^{\mathrm{ns}}$ & $1.3^{* * * *}$ & $1.4^{* *}$ & $1.0^{\mathrm{ns}}$ & $0.0^{\mathrm{ns}}$ & $100.0^{\mathrm{ns}}$ & $6.8^{* * *}$ & $2.1^{* * *}$ & $31.0^{\mathrm{ns}}$ \\
\hline Mkwanyule & $1.2^{\mathrm{ns}}$ & $1.2^{* * *}$ & $1.6^{\mathrm{ns}}$ & $1.2^{\mathrm{ns}}$ & $1.6^{\mathrm{ns}}$ & $99.3^{\mathrm{ns}}$ & $13.1^{* * *}$ & $3.8^{\mathrm{ns}}$ & $22.6^{*}$ \\
\hline Mnacho & $1.2^{\mathrm{ns}}$ & $1.6^{\mathrm{ns}}$ & $1.4^{*}$ & $1.1^{\mathrm{ns}}$ & $2.0^{\mathrm{ns}}$ & $97.4^{\mathrm{ns}}$ & $16.2^{*}$ & $4.9^{\mathrm{ns}}$ & $31.1^{\mathrm{ns}}$ \\
\hline Mnondodya & $1.4^{\mathrm{ns}}$ & $2.0^{\mathrm{ns}}$ & $1.6^{\mathrm{ns}}$ & $3.1^{* * * *}$ & $59.8^{* * *}$ & $41.5^{* * *}$ & $11.8^{* * *}$ & $4.2^{\mathrm{ns}}$ & $30.8^{\mathrm{ns}}$ \\
\hline Mombasa & $1.0^{\mathrm{ns}}$ & $1.0^{* * *}$ & $1.5^{*}$ & $1.2^{\mathrm{ns}}$ & $10.0^{* * *}$ & $92.6^{\mathrm{ns}}$ & $22.5^{\mathrm{ns}}$ & $5.2^{\mathrm{ns}}$ & $32.6^{* *}$ \\
\hline Mreteta & $1.2^{\mathrm{ns}}$ & $2.2^{\mathrm{ns}}$ & $2.0^{\mathrm{ns}}$ & $1.1^{\mathrm{ns}}$ & $2.3^{\mathrm{ns}}$ & $98.6^{\mathrm{ns}}$ & $22.8^{\mathrm{ns}}$ & $5.9^{\mathrm{ns}}$ & $31.6^{\mathrm{ns}}$ \\
\hline Musa Said & $1.0^{\mathrm{ns}}$ & $1.6^{\mathrm{ns}}$ & $2.0^{\mathrm{ns}}$ & $1.2^{\mathrm{ns}}$ & $1.6^{\mathrm{ns}}$ & $99.0^{\mathrm{ns}}$ & $26.9^{\text {ns }}$ & $4.8^{\mathrm{ns}}$ & $29.8^{\text {ns }}$ \\
\hline Mweda & $1.3^{\mathrm{ns}}$ & $1.5^{\mathrm{ns}}$ & $1.6^{\mathrm{ns}}$ & $1.2^{\mathrm{ns}}$ & $5.3^{\mathrm{ns}}$ & $97.8^{\mathrm{ns}}$ & $35.0^{* * *}$ & $6.8^{\mathrm{ns}}$ & $31.2^{\mathrm{ns}}$ \\
\hline Mwendowaloya & $1.0^{\mathrm{ns}}$ & $1.3^{* * *}$ & $1.5^{*}$ & $1.2^{\mathrm{ns}}$ & $7.2^{* *}$ & $99.1^{\mathrm{ns}}$ & $10.1^{* * *}$ & $5.5^{\mathrm{ns}}$ & $32.3^{*}$ \\
\hline Mzigowamwizi & $1.2^{\mathrm{ns}}$ & $1.2^{* * * *}$ & $1.8^{\mathrm{ns}}$ & $1.9^{* * * *}$ & $17.3^{* * *}$ & $80.8^{* * *}$ & $15.3^{* *}$ & $4.3^{\mathrm{ns}}$ & $27.2^{\mathrm{ns}}$ \\
\hline Nachinyaya & $2.4^{* * * *}$ & $2.1^{\mathrm{ns}}$ & $2.2^{\mathrm{ns}}$ & $1.2^{\mathrm{ns}}$ & $4.1^{\mathrm{ns}}$ & $98.6^{\mathrm{ns}}$ & $14.9^{* *}$ & $5.5^{\mathrm{ns}}$ & $24.5^{\mathrm{ns}}$ \\
\hline Nakuchima & $1.0^{\mathrm{ns}}$ & $1.6^{\mathrm{ns}}$ & $1.7^{\mathrm{ns}}$ & $1.5^{* * *}$ & $6.4^{* *}$ & $94.9^{\text {ns }}$ & $8.4^{* * *}$ & $3.9^{\text {ns }}$ & $34.3^{* * *}$ \\
\hline Nalilekuchumba & $1.4^{\mathrm{ns}}$ & $1.9^{\mathrm{ns}}$ & $1.7^{\mathrm{ns}}$ & $1.5^{* * *}$ & $14.3^{* * *}$ & $91.1^{*}$ & $13.6^{* * *}$ & $4.5^{\mathrm{ns}}$ & $24.2^{\mathrm{ns}}$ \\
\hline Namanjongonda & $2.0^{*}$ & $2.0^{\mathrm{ns}}$ & $2.2^{\mathrm{ns}}$ & $1.7^{* * * *}$ & $29.0^{* * *}$ & $73.8^{* * *}$ & $17.3^{\mathrm{ns}}$ & $4.6^{\mathrm{ns}}$ & $32.5^{* *}$ \\
\hline Namkola & $1.5^{\mathrm{ns}}$ & $1.7^{\mathrm{ns}}$ & $1.4^{* *}$ & $1.1^{\mathrm{ns}}$ & $3.8^{\mathrm{ns}}$ & $97.9^{\text {ns }}$ & $14.8^{* * *}$ & $5.9^{\text {ns }}$ & $27.4^{\mathrm{ns}}$ \\
\hline Nanjeja & $2.0^{* *}$ & $2.4^{\mathrm{ns}}$ & $2.8^{\mathrm{ns}}$ & $1.6^{* * * *}$ & $23.7^{* * *}$ & $74.4^{* * *}$ & $13.8^{* * * *}$ & $3.9^{\text {ns }}$ & $35.8^{* * * *}$ \\
\hline
\end{tabular}




\begin{tabular}{|c|c|c|c|c|c|c|c|c|c|}
\hline Nanjenjeha & $1.1^{\mathrm{ns}}$ & $2.1^{\mathrm{ns}}$ & $1.9^{\mathrm{ns}}$ & $1.3^{\mathrm{ns}}$ & $6.1^{*}$ & $96.7^{\mathrm{ns}}$ & $19.6^{\mathrm{ns}}$ & $4.6^{\mathrm{ns}}$ & $25.9^{\mathrm{ns}}$ \\
\hline Ndanda & $1.5^{\mathrm{ns}}$ & $1.8^{\mathrm{ns}}$ & $1.6^{\mathrm{ns}}$ & $1.0^{\mathrm{ns}}$ & $0.0^{\mathrm{ns}}$ & $100.0^{\mathrm{ns}}$ & $17.2^{\mathrm{ns}}$ & $4.5^{\mathrm{ns}}$ & $28.5^{\mathrm{ns}}$ \\
\hline Nkutiao & $1.0^{\mathrm{ns}}$ & $1.1^{* * *}$ & $1.5^{* *}$ & $1.1^{\mathrm{ns}}$ & $0.6^{\mathrm{ns}}$ & $99.6^{\mathrm{ns}}$ & $7.5^{* * *}$ & $6.6^{\mathrm{ns}}$ & $31.8 *$ \\
\hline Ntara & $1.2^{\mathrm{ns}}$ & $2.7^{*}$ & $1.6^{\mathrm{ns}}$ & $1.1^{\mathrm{ns}}$ & $1.0^{\mathrm{ns}}$ & $99.0^{\mathrm{ns}}$ & $25.8^{\mathrm{ns}}$ & $5.2^{\mathrm{ns}}$ & $25.1^{\mathrm{ns}}$ \\
\hline Ntonto & $2.5^{* * *}$ & $2.8^{* * *}$ & $2.8^{\mathrm{ns}}$ & $2.7^{* * * *}$ & $57.1^{* * *}$ & $42.6^{* * *}$ & $31.3^{* * *}$ & $4.5^{\mathrm{ns}}$ & $25.4^{\mathrm{ns}}$ \\
\hline Nyankagile & $2.4^{* * *}$ & $2.7^{* *}$ & $2.4^{\mathrm{ns}}$ & $1.1^{\mathrm{ns}}$ & $1.2^{\mathrm{ns}}$ & $99.4^{\mathrm{ns}}$ & $15.1^{* *}$ & $4.4^{\mathrm{ns}}$ & $28.8^{\mathrm{ns}}$ \\
\hline Nyoka & $1.8^{\mathrm{ns}}$ & $2.6^{*}$ & $1.9^{\mathrm{ns}}$ & $1.0^{\mathrm{ns}}$ & $0.0^{\mathrm{ns}}$ & $100.0^{\mathrm{ns}}$ & $21.9^{\mathrm{ns}}$ & $6.3^{\mathrm{ns}}$ & $32.8^{* *}$ \\
\hline Sakada & $1.5^{\mathrm{ns}}$ & $1.5^{*}$ & $1.4^{*}$ & $1.2^{\mathrm{ns}}$ & $3.3^{\mathrm{ns}}$ & $98.7^{\mathrm{ns}}$ & $6.5^{* * *}$ & $2.1^{* * * *}$ & $25.8^{\mathrm{ns}}$ \\
\hline Salanga & $1.2^{\mathrm{ns}}$ & $2.0^{\mathrm{ns}}$ & $1.4^{* *}$ & $1.0^{\mathrm{ns}}$ & $0.6^{\mathrm{ns}}$ & $100.0^{\mathrm{ns}}$ & $18.4^{\mathrm{ns}}$ & $5.2^{\mathrm{ns}}$ & $31.7^{\mathrm{ns}}$ \\
\hline Sheria & $1.4^{\mathrm{ns}}$ & $1.8^{\mathrm{ns}}$ & $1.8^{\mathrm{ns}}$ & $2.3^{* * *}$ & $42.7^{* * *}$ & $54.5^{* * *}$ & $7.7^{* * *}$ & $3.2^{* * *}$ & $22.2^{*}$ \\
\hline Simanyu & $1.3^{\mathrm{ns}}$ & $1.4^{*}$ & $1.7^{\mathrm{ns}}$ & $1.2^{\mathrm{ns}}$ & $3.9^{\mathrm{ns}}$ & $97.7^{\mathrm{ns}}$ & $23.9^{\mathrm{ns}}$ & $7.0^{\mathrm{ns}}$ & $31.9^{*}$ \\
\hline Sumu ya Panya & $1.7^{\mathrm{ns}}$ & $1.8^{\mathrm{ns}}$ & $2.0^{\mathrm{ns}}$ & $2.1^{* * *}$ & $46.2^{* * *}$ & $68.2^{* * *}$ & $23.9^{\text {ns }}$ & $5.0^{\mathrm{ns}}$ & $24.4^{\mathrm{ns}}$ \\
\hline Supa & $1.2^{\mathrm{ns}}$ & $1.9^{\mathrm{ns}}$ & $2.1^{\mathrm{ns}}$ & $1.0^{\mathrm{ns}}$ & $1.3^{\mathrm{ns}}$ & $99.6^{\mathrm{ns}}$ & $19.5^{\mathrm{ns}}$ & $3.4^{* *}$ & $29.6^{\mathrm{ns}}$ \\
\hline Supa B & $1.1^{\mathrm{ns}}$ & $1.3^{* * *}$ & $1.4^{* *}$ & $1.0^{\mathrm{ns}}$ & $0.0^{\mathrm{ns}}$ & $100.0^{\mathrm{ns}}$ & $24.9^{\mathrm{ns}}$ & $6.1^{\mathrm{ns}}$ & $38.4^{* * *}$ \\
\hline Supa Jangwa & $1.0^{\mathrm{ns}}$ & $1.4^{* *}$ & $1.5^{*}$ & $1.1^{\mathrm{ns}}$ & $1.6^{\mathrm{ns}}$ & $99.9^{\text {ns }}$ & $20.7^{\mathrm{ns}}$ & $4.4^{\mathrm{ns}}$ & $32.2^{*}$ \\
\hline Vicent & $1.5^{\mathrm{ns}}$ & $1.7^{\mathrm{ns}}$ & $2.1^{\mathrm{ns}}$ & $1.0^{\mathrm{ns}}$ & $0.0^{\mathrm{ns}}$ & $100.0^{\mathrm{ns}}$ & $21.8^{\mathrm{ns}}$ & $5.0^{\mathrm{ns}}$ & $30.4^{\mathrm{ns}}$ \\
\hline Victory & $1.3^{\mathrm{ns}}$ & $2.5^{\mathrm{ns}}$ & $2.0^{\mathrm{ns}}$ & $1.4^{* * * *}$ & $30.6^{* * *}$ & $86.5^{* * *}$ & $15.4^{* *}$ & $5.0^{\mathrm{ns}}$ & $27.8^{\mathrm{ns}}$ \\
\hline Mean & 1.4 & 1.8 & 1.9 & 1.4 & 14.3 & 89.7 & 16.5 & 4.7 & 29.7 \\
\hline Mean (2014) & 1.4 & 1.9 & 2.0 & 1.5 & 14.5 & 91.0 & 18.7 & 4.8 & 27.1 \\
\hline Mean (2015) & 1.4 & 1.7 & 1.7 & 1.3 & 14.1 & 88.5 & 14.0 & 4.5 & 32.4 \\
\hline $\mathrm{CV}$ & 20.0 & 15.2 & 18.4 & 11.6 & 21.5 & 4.8 & 18.2 & 17.2 & 7.7 \\
\hline
\end{tabular}

${ }^{\mathrm{ns}} \mathrm{P}>0.05, * \mathrm{P} \leq 0.05, * * \mathrm{P} \leq 0.01$, and $* * * \mathrm{P} \leq 0.001$

Mean (2014), Mean (2015) - means for cropping season one and two, respectively.

$\mathrm{CV}$ - coefficient of variation. 
Similar results were observed for root necrosis incidence. Kiroba had low mean root necrosis incidence of $0.0 \%$ as none of the roots showed any disease. Other landraces including Azoa, Bangi, Benny, Chidubwa, Chimaje, Katewanya, Limbanga, Mfaransa, and Nachinyaya also had low mean root necrosis incidence ranging from 0.0 to $5.5 \%$, and they were not significantly different $(\mathrm{P} \leq 0.05)$ from Kiroba. Albert had the highest mean root necrosis incidence of $99.2 \%$. Landraces including Cosmas, Kalinda, Kigoma Red, Mdimbe, Mnondodya, Nanjenjeha, and Ntonto had significantly higher mean root necrosis incidence than Kiroba that ranged from 6.1 to $89.8 \%$ (Table 1). Kiroba exhibited the least root necrosis symptoms and therefore all roots $(100 \%)$ were usable. Other landraces with high amounts of usable roots that were not significantly different from the usable roots in Kiroba included: Azoa, Benny, Mweda, Nakuchima and Simanyu (all >95\%), and Chimaje, Kiwinda and Limbanga (all 100\%) (Table 1). Seriously affected landraces with lower amounts of usable roots included Mnondodya 41.5\%, Ntonto 42.6\%, Sheria 54.5\%, Kigoma red 37.6\%, Sumu ya Panya $68.2 \%$, and Cosmas $69.9 \%$, which were all comparable to the susceptible control Albert 53.2\%.

\subsection{Cassava yield traits}

The highest mean root weight was recorded in the landrace Mweda at 35.0 tonnes per hectare $(\mathrm{t} / \mathrm{ha})$, which was significantly $(\mathrm{P} \leq 0.001)$ higher than that of Kiroba $22.4 \mathrm{t} /$ ha (Table 1$)$. Kiroba's root weight was not significantly different from other landraces except Binti Juma, Cosmas, Hamad Rashid, Mwendo wa Loya, Nkutiao, Sakada, and Sheria, which had significantly $(\mathrm{P} \leq 0.05)$ low root weight ranging between 4.7 to $15.8 \mathrm{t} / \mathrm{ha}$ (Table 1$)$. The highest mean number of roots per plant was recorded in Simanyu at 7.0, which was not significantly different from Kiroba at 5.4 (Table 1). Kiroba's mean number of roots per plant was not significantly different from that of other landraces apart from Kikombe, Kifuu cha Nazi, Kiwinda, Mfaransa, Nakuchima, Sakada, Sheria, and Supa, which had significantly (P $\leq 0.05$ ) lower mean number of roots ranging between 2.1 to 3.6 (Table 1). The highest dry matter content was recorded in Supa B 38.4\%, Binamuli 37.3\%, and Liumbukwa 36.4\%. Additionally, these landraces had significantly $(\mathrm{P} \leq 0.001)$ higher dry matter content compared to Kiroba $27.2 \%$ (Table 1). Significantly low dry matter content was recorded in Sheria 22.2\% and Mkwanyule 22.6\%. 


\subsection{Mean squares and sum of squares for traits evaluated}

The ANOVA revealed a larger percentage of total sum of squares (SS) ranging from 42.2 to $78.2 \%$ attributed to the cassava genotypes for CBSD foliar symptoms at 3,6 and 9 MAP; root necrosis severity; root necrosis incidence; usable roots; root weight; number of roots per plant and dry matter content. The mean squares were also very highly significant $(\mathrm{P} \leq 0.001)$ (Tables 2, 3, and 4). Genotype by season interaction was second with SS percentage ranging from 20.9 to $35.1 \%$ and with very highly significant $(\mathrm{P} \leq 0.001)$ mean squares (Tables 2,3 , and 4). The environment accounted for the least variation observed with SS ranging from 0.01 to $26.8 \%$ but still with very highly significant mean squares $(\mathrm{P} \leq 0.001)$. All the above differences were analysed for their effect on CBSD leaf symptoms severity at 6 and 9 MAP, root necrosis severity, usable roots, root weight, dry matter content, and harvest index (Tables 2, 3, and 4). A large SS indicated that the genotypes were diverse with large differences among the means, contributing to most variations in the traits analysed. The smaller proportion of SS for genotype by environment interaction indicated that the differences among the genotypes by environment interaction means was not very high. The magnitude of only the environment SS was very small thus indicating that effect of the environment on two seasons was not that substantial. 
Table 2

Means of squares and sums of squares for CBSD foliar symptoms.

\begin{tabular}{l|l|lll|lll|lll|}
\hline Source of Variation & df & \multicolumn{3}{|l|}{ Foliar symptoms at 3 MAP } & \multicolumn{3}{|l|}{ Foliar symptoms at 6 MAP } & \multicolumn{3}{l|}{ Foliar symptoms at 9 MAP } \\
\cline { 3 - 12 } & & MS & SS & SS (\%) & MS & SS & SS (\%) & MS & SS & SS (\%) \\
\hline Total & 173 & - & 89.9 & - & - & 143.9 & - & - & 127.6 & - \\
Season & 1 & $0.03^{\text {ns }}$ & 0.03 & 0.03 & $2.74^{* * *}$ & 2.74 & 1.90 & $7.45^{* * *}$ & 7.45 & 5.84 \\
Block (Season*Replicate) & 46 & $0.11^{\text {ns }}$ & 5.01 & 5.57 & $0.10^{\text {ns }}$ & 4.39 & 3.05 & $0.17^{*}$ & 7.82 & 6.13 \\
Genotype & 63 & $1.05^{* * *}$ & 66.1 & 73.5 & $1.41^{* * *}$ & 88.8 & 61.7 & $1.07^{* * *}$ & 67.5 & 52.9 \\
Genotype*Season & 63 & $0.30^{* * *}$ & 18.8 & 20.9 & $0.76^{* * *}$ & 48.0 & 33.4 & $0.71^{* * *}$ & 44.8 & 35.1 \\
Error & 210 & 0.08 & 17.3 & - & 0.08 & 16.3 & - & 0.12 & 24.5 & - \\
\hline
\end{tabular}

df, MS, SS - degrees of freedom, mean squares, and sum of squares, respectively

${ }^{\text {ns }} \mathrm{P}>0.05,{ }^{*} \mathrm{P} \leq 0.05, * * \mathrm{P} \leq 0.01$, and ${ }^{* * *} \mathrm{P} \leq 0.001$

Table 3

Means of squares and sums of squares for CBSD root symptoms.

\begin{tabular}{|c|c|c|c|c|c|c|c|c|c|c|}
\hline \multirow[t]{2}{*}{ Source of Variation } & \multirow[t]{2}{*}{ Df } & \multicolumn{3}{|c|}{ Root necrosis severity } & \multicolumn{3}{|c|}{ Root necrosis incidence } & \multicolumn{3}{|c|}{ Usable roots } \\
\hline & & MS & SS & SS (\%) & MS & SS & SS $(\%)$ & MS & SS & SS $(\%)$ \\
\hline Total & 173 & - & 145.3 & - & - & 193000.9 & - & - & 111137.8 & - \\
\hline Season & 1 & $5.20^{* * *}$ & 5.20 & 3.58 & $21.6^{\mathrm{ns}}$ & 21.6 & 0.01 & $573.8^{* * *}$ & 573.8 & 0.52 \\
\hline Block (Season*Replicate) & 46 & $0.02^{\mathrm{ns}}$ & 1.10 & 0.76 & $11.8^{\mathrm{ns}}$ & 540.9 & 0.28 & $23.6^{\mathrm{ns}}$ & 1083.7 & 0.98 \\
\hline Genotype & 63 & $1.69^{* * * *}$ & 106.3 & 73.2 & $2396.9^{* * *}$ & 151002.3 & 78.2 & $1340.1^{* * * *}$ & 84425.4 & 76.0 \\
\hline Season*Genotype & 63 & $0.52^{* * *}$ & 32.7 & 22.5 & $657.7^{* * *}$ & 41436.1 & 21.5 & $397.7^{* * *}$ & 25054.9 & 22.5 \\
\hline Error & 210 & 0.03 & 5.60 & - & 9.4 & 1981.1 & - & 18.6 & 3895.6 & - \\
\hline
\end{tabular}

df, MS, SS - degrees of freedom, mean squares, and sum of squares, respectively

${ }^{\text {ns }} \mathrm{P}>0.05, * \mathrm{P} \leq 0.05, * * \mathrm{P} \leq 0.01$, and $* * * \mathrm{P} \leq 0.001$ 
Table 4

Means of squares and sums of squares for cassava root traits.

\begin{tabular}{|c|c|c|c|c|c|c|c|c|c|c|}
\hline \multirow[t]{2}{*}{ Source of Variation } & \multirow[t]{2}{*}{ Df } & \multicolumn{3}{|c|}{ Root weight (t/ha) } & \multicolumn{3}{|c|}{ Root number } & \multicolumn{3}{|c|}{ Dry matter content } \\
\hline & & MS & SS & SS (\%) & MS & SS & SS (\%) & MS & SS & SS (\%) \\
\hline Total & 173 & - & 20294.5 & - & - & 567.0 & - & - & 9937.2 & - \\
\hline Season & 1 & $1936.1^{\text {**** }}$ & 1936.1 & 9.54 & $6.68^{\mathrm{ns}}$ & 6.68 & 1.18 & $2665.1^{* * *}$ & 2665.1 & 26.8 \\
\hline Block (Season*Replicate) & 46 & $7.20^{\mathrm{ns}}$ & 331.3 & 1.63 & $0.72^{\mathrm{ns}}$ & 33.1 & 5.84 & $6.72^{\mathrm{ns}}$ & 309.1 & 3.11 \\
\hline Genotype & 63 & $194.0^{* * *}$ & 12218.6 & 60.2 & $5.73^{* * * *}$ & 360.7 & 63.6 & $66.8^{* * *}$ & 4209.1 & 42.4 \\
\hline Season*Genotype & 63 & $92.2^{* * * *}$ & 5808.5 & 28.6 & $2.64^{* * * *}$ & 166.5 & 29.4 & $43.7^{* * *}$ & 2753.9 & 27.7 \\
\hline Error & 210 & 9.0 & 1898.2 & - & 0.65 & 136.0 & - & 5.23 & 1098.4 & - \\
\hline
\end{tabular}

$\mathrm{df}, \mathrm{MS}$, SS - degrees of freedom, mean squares, and sum of squares, respectively

${ }^{\mathrm{ns}} \mathrm{P}>0.05, * \mathrm{P} \leq 0.05, * * \mathrm{P} \leq 0.01$, and $* * * \mathrm{P} \leq 0.001$ 


\subsection{Environmental influences on traits tested}

In this study data for foliar symptoms was collected in March (3 MAP), June (6 MAP) and September (9 MAP) while data for root symptoms, root weight, dry matter content, and harvest index were collected in December after harvesting. It was generally observed that higher temperatures, relative humidity, and rainfall resulted to a period of active growth in the plants with reduced CBSD symptom severity. Rainfall, relative humidity and temperatures were higher at 3 MAP when compared to 6 MAP and 9 MAP (Fig. 3). This could explain the drastic increase in mean leaf CBSD symptoms severity from 3 MAP to 6 MAP but a slight increase in symptoms severity from 6 MAP to 9 MAP. Additionally, higher means for CBSD foliar and root symptoms were observed in trial 1 in 2014 compared to trial 2 in 2015 (Table 1). Slightly higher rainfall and temperatures were recorded in 2015 compared to 2014 during 1 to 9 MAP (Fig. 3), and this may have influenced the lower symptom severity observed in trial two (Table 1). Season effect was also observed in root weight, number of roots per plant, and dry matter content. There was a higher mean root weight of $18.7 \mathrm{t} /$ ha in 2014 when compared to $14.0 \mathrm{t} / \mathrm{ha}$ in 2015 . In contrast, the mean dry matter content was $27.1 \%$ in 2014 and $32.4 \%$ in 2015 (Table 1). The high levels of rainfall recorded in November $132.2 \mathrm{~mm}$ and December $102.9 \mathrm{~mm}$ in 2014 (Fig. 3), may have influenced higher root weight, but a lower dry matter content.

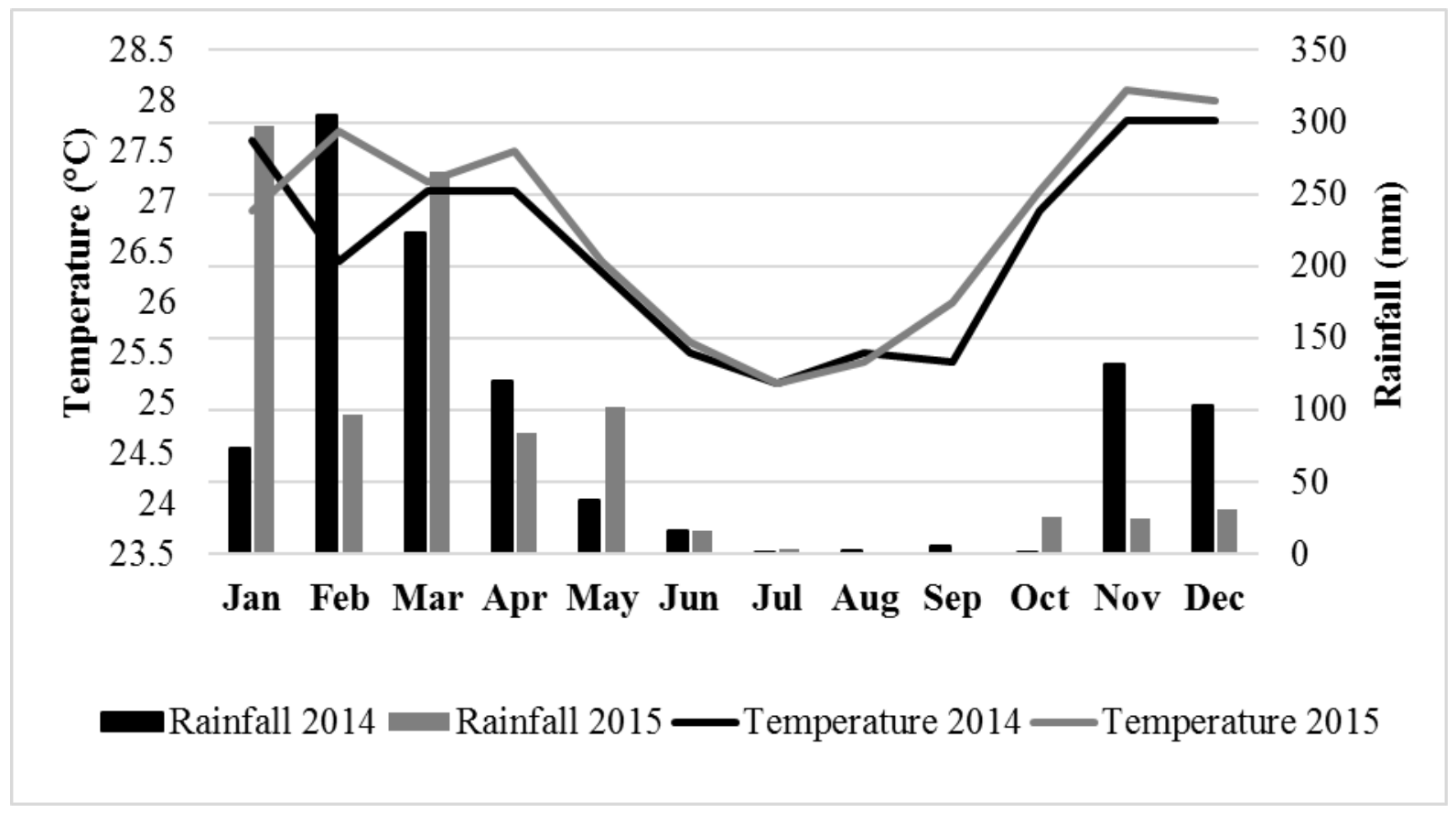

Fig. 3. Seasonal variability in rainfall and temperature. 


\subsection{Correlation analysis}

There was a significant positive correlation between CBSD foliar symptoms severity at 3 MAP and 6 MAP $(\mathrm{P} \leq 0.001, \mathrm{r}=0.54$ ) (Table 5). A significant moderately positive correlation was observed between foliar symptoms severity at 3 MAP and 9 MAP $(\mathrm{P} \leq 0.001$, $r=0.43$ ). Similarly, a significant positive correlation was observed between foliar symptoms severity at $6 \mathrm{MAP}$ and $9 \mathrm{MAP}(\mathrm{P} \leq 0.001, \mathrm{r}=0.61)$. These results showed that approximately $50 \%$ of the plants with foliar symptoms at 3 MAP also had symptoms at 6 and 9 MAP.

No correlation was observed between foliar symptoms at $3 \mathrm{MAP}$ and root necrosis severity (Table 5). However, there was a significant positive correlation between foliar symptoms at 3 MAP and root necrosis severity $(\mathrm{P} \leq 0.05, \mathrm{r}=0.11)$, between foliar symptoms at 6 MAP and root necrosis severity $(\mathrm{P} \leq 0.001, \mathrm{r}=0.22)$, and between foliar symptoms at 9 MAP and root necrosis $(\mathrm{P} \leq 0.001, \mathrm{r}=0.22)$. Similar results were observed between symptoms and root necrosis incidence where, foliar symptoms at 3 MAP was positively correlated to root necrosis incidence $(\mathrm{P} \leq 0.01, \mathrm{r}=0.13)$, foliar symptoms at $6 \mathrm{MAP}$ was positively correlated to root necrosis incidence $(\mathrm{P} \leq 0.001, \mathrm{r}=0.21)$, and foliar symptoms at 9 MAP was positively correlated to root necrosis incidence $(\mathrm{P} \leq 0.001, \mathrm{r}=0.20)$ (Table 5). Additionally, a significantly high positive correlation was found between root necrosis and root necrosis incidence $(\mathrm{P} \leq 0.001, \mathrm{r}=0.90)$.

On the other hand, a significant low negative correlation was observed between foliar symptoms and usable roots at $3 \mathrm{MAP}(\mathrm{P} \leq 0.001, \mathrm{r}=-0.15), 6 \mathrm{MAP}(\mathrm{P} \leq 0.001, \mathrm{r}=-0.23)$ and 9 MAP $(\mathrm{P} \leq 0.001, \mathrm{r}=-0.22)$. A highly negative correlation was observed between root necrosis and usable roots $(\mathrm{P} \leq 0.001, \mathrm{r}=-0.88)$, as well as root necrosis incidence and usable roots $(\mathrm{P} \leq 0.001, \mathrm{r}=-0.88)$. The low positive correlation between foliar and root symptoms showed that plants with foliar symptoms did not necessarily have root symptoms. The results also showed that plants with severe root necrosis also had high root necrosis incidence and subsequent low amount of usable roots.

Finally, a significant negative correlation was observed between root weight and root necrosis incidence $(\mathrm{P} \leq 0.001, \mathrm{r}=-0.14)$ (Table 5). Comparably, a negative correlation was observed between foliar symptoms at $6 \mathrm{MAP}$ and dry matter content $(\mathrm{P} \leq 0.001, \mathrm{r}=-0.15)$, 
foliar symptoms at 9 MAP and dry matter content $(\mathrm{P} \leq 0.001, \mathrm{r}=-0.15)$, and root necrosis and dry matter content $(\mathrm{P} \leq 0.001, \mathrm{r}=-0.15)$. A high positive correlation was recorded between root weight and number of roots per plant $(\mathrm{P} \leq 0.001 \mathrm{r}=0.58)$. Simillarly, a positive correlation was observed between root weight and usable roots $(\mathrm{P} \leq 0.01 \mathrm{r}=0.13)$. This showed that some high yielding landraces had more usable roots (Table 5). Although there were low negative correlations between CBSD symptoms and number of roots per plant, they were nonetheless not significant. 


\section{Table 5}

Correlation coefficient values for CBSD symptoms and root traits

\begin{tabular}{|c|c|c|c|c|c|c|c|c|c|}
\hline & $\begin{array}{l}\text { Foliar } \\
\text { symptoms } \\
\text { at } 3 \mathrm{MAP}\end{array}$ & $\begin{array}{l}\text { Foliar } \\
\text { symptoms } \\
\text { at } 6 \text { MAP }\end{array}$ & $\begin{array}{l}\text { Foliar } \\
\text { symptoms } \\
\text { at } 9 \text { MAP }\end{array}$ & $\begin{array}{l}\text { Root } \\
\text { necrosis }\end{array}$ & $\begin{array}{l}\text { Root necrosis } \\
\text { incidence }(\%)\end{array}$ & $\begin{array}{l}\text { Usable } \\
\text { roots (\%) }\end{array}$ & $\begin{array}{l}\text { Root } \\
\text { weight } \\
\text { (t/ha) }\end{array}$ & $\begin{array}{l}\text { Root } \\
\text { number }\end{array}$ & $\begin{array}{l}\text { Dry matter } \\
\text { content } \\
(\%)\end{array}$ \\
\hline Foliar symptoms at 3 MAP & 1 & $.54^{* * * *}$ & $.43^{* * * *}$ & $.11^{*}$ & $.13^{* *}$ & $-.15^{* * *}$ & $-.08^{\mathrm{ns}}$ & $-.03^{\mathrm{ns}}$ & $-.05^{\mathrm{ns}}$ \\
\hline Foliar symptoms at $6 \mathrm{MAP}$ & & 1 & $.61^{* * *}$ & $.22^{* * *}$ & $.21^{* * *}$ & $-.23^{* * *}$ & $.04^{\mathrm{ns}}$ & $-.01^{\mathrm{ns}}$ & $-.15^{* * *}$ \\
\hline Foliar symptoms at 9 MAP & & & 1 & $.22^{* * * *}$ & $.20^{* * * *}$ & $-.22^{* * * *}$ & $.06^{\mathrm{ns}}$ & $-.01^{\mathrm{ns}}$ & $-.15^{* * *}$ \\
\hline Root necrosis & & & & 1 & $.90^{* * * *}$ & $-.88^{* * *}$ & $-.07^{\mathrm{ns}}$ & $.01^{\mathrm{ns}}$ & $-.15^{* * *}$ \\
\hline Root necrosis incidence $(\%)$ & & & & & 1 & $-.88^{* * * *}$ & $-.14^{* *}$ & $-.02^{\mathrm{ns}}$ & $-.07^{\mathrm{ns}}$ \\
\hline Usable roots $(\%)$ & & & & & & 1 & $.12^{*}$ & $.02^{\mathrm{ns}}$ & $.06^{\mathrm{ns}}$ \\
\hline Root weight (t/ha) & & & & & & & 1 & $.58^{* * * *}$ & $-.10^{*}$ \\
\hline Root number & & & & & & & & 1 & $.01^{\mathrm{ns}}$ \\
\hline Dry matter content $(\%)$ & & & & & & & & & 1 \\
\hline
\end{tabular}

${ }^{n s} \mathrm{P}>0.05, * \mathrm{P} \leq 0.05, * * \mathrm{P} \leq 0.01$, and $* * * \mathrm{P} \leq 0.001$ 


\section{Discussion}

Varied responses to CBSD were recorded among the tested landraces in field experiments for two seasons. In the case of resistant plants, infection by viruses can occur but pathogen growth and disease development in the plant was minimal. Consequently, the disease symptoms were generally localized or absent (Cooper and Jones, 1983; Kang et al., 2005). These were the characteristics seen on Namikonga which had perpetually exhibited no or low symptoms severity for many years, and hence considered resistant (Maruthi et al., 2014; Masumba et al., 2017). The term tolerance is used to describe a host that can be infected by a virus which causes symptoms without significantly diminishing the plant growth or yield (Cooper and Jones, 1983). An example in our case is Kiroba, the tolerant control, which had foliar symptoms severity score of up to 2 , but no visible root symptoms, and thus had $100 \%$ usable roots. Susceptibility on the other hand describes a host plant with high virus titres, severe symptoms both on leaves and roots and thus significant yield loss (Maruthi et al., 2014). We used Albert as the susceptible control, which expressed both leaf and root symptoms, and as a result reduced usable roots. Using these criteria, we classified the cassava landraces into the resistant, tolerant and susceptible categories.

Our resistant landraces had minimal foliar and root symptoms with $100 \%$ usable roots. They included Chimaje, Chipanda, Limbanga, Mfaransa, Mkwanyule, Mweda, Mwendo wa Loya, Ndanda, Nkutiao, Sakada, Supa B, and Supa Jangwa. The tolerant landraces included Chidubwa, Kikwada, Mbuyu, Mreteta, Musa Said, Nachinyaya, Nanjenjeha, Nyankagile, Nyoka, and Vicent. These readily developed foliar symptoms but with delayed or absent root symptoms, similar to Kiroba (Hillocks and Jennings, 2003). Other landraces which can also be regarded as tolerant but had slightly higher foliar and root symptoms compared to Kiroba included Binty Ally, Kalinda, Kibangameno, Kifuu cha Nazi, Kitumbua, Likonde II, Mdimbe, Namanjongonda, Nalilekuchumba, Nanjeja, and Victory. The susceptible landraces included Cosmas, Kigoma Red, Mnondodya, Ntonto, Sheria, and Sumu ya Panya. These were similar to susceptible control Albert, which had moderate to severe foliar symptom severity, but high root symptoms severity and only about half the roots were usable. The low foliar symptom severity observed in Albert showed that this probably is not the most susceptible variety and has some levels of tolerance to the disease for foliar symptoms, but not to root necrosis, which will only be discovered when the plants are harvested. This is the greatest 
source of food insecurity to cassava farmers growing CBSD-susceptible varieties as the extent of the damage is only visible after harvest.

Genotype and genotype by environment interactions contributed to major variations observed for all traits tested. They accounted for the largest SS observed with very highly significant means squares. The environment alone accounted for the smallest SS except for a few genotypes. The results showed that although the traits examined were mostly under genetic control, there were a few genotypes whose trait expression was influenced by the environment. Similar results were reported earlier on genotype and genotype by environment effects on CBSD symptom expression and root weight (Boakye et al., 2013; Tumuhimbise et al., 2014; Pariyo et al., 2015). High disease incidences and severe symptoms have been reported in higher altitudes prone to cooler temperatures, during low night temperatures and moisture stress (Nichols, 1950; Jennings, 1957; Rwegasira, 2009). CBSD symptoms are usually variable and irregular and depend on many factors including plant age, cultivar (genotype), environmental conditions (i.e. altitude, temperature, rainfall quantity) and virus species (Hillocks and Jennings, 2003; Mohammed et al., 2012; Patil et al., 2014). We generally observed active plant growth with concurrent reduction in disease severity at higher temperatures, relative humidity and rainfall.

The low positive correlation of $r<0.25$ between leaf symptoms, and both root necrosis and incidence indicated that the presence of leaf symptoms does not always determine the presence of root necrosis as observed in landraces Kikwada and Kiroba. They readily expressed leaf symptoms but with low or no root necrosis. These observations are in agreement with Nzuki et al. (2017) who reported different QTLs for root necrosis and foliar symptoms in Kiroba. They identified 15 significant QTLs, two were associated with CBSD root necrosis only, while seven were associated with CBSD foliar symptoms only. These results are also similar to $<50 \%$ association between foliar symptoms and root necrosis reported earlier (Hillocks et al., 1996; Abaca et al., 2012). However, high positive correlation ( $\mathrm{P} \leq 0.001, r=0.90)$ was seen between root necrosis severity and incidence. This meant that varieties with high root necrosis severity also had high root necrosis incidences and consequently greater reduction in usable roots. CBSD symptoms both on leaves and roots can also affect key agronomic traits such as root weight and dry matter content (Rwegasira, 2009). The total root weight and number of roots per plant were mostly negatively correlated 
with CBSD symptoms although the correlation was not statistically significant. However, there was a significant negative correlation $(\mathrm{P} \leq 0.001, \mathrm{r}=-0.14)$ between total root weight and root necrosis incidences indicating that a high root necrosis severity can lead to severely reduced root weight. Some roots with high root necrosis severity may also be constricted or deformed, making them difficult to process, further resulting in reduced root weight (Legg and Hillocks, 2003). A significantly negative correlation between CBSD symptoms severity (on leaves and roots) and dry matter content showed that the disease causes reduction to cassava profitability. Similar results were also reported previously (Aigbe and Remisson, 2010; Abaca et al., 2012).

In addition to disease resistance or tolerance, some landraces also had desirable root traits. Mweda, Limbanga, and Chipanda, which were resistant to CBSD, also had high root weight, high number of roots per plant, and high dry matter content. The tolerant control Kiroba had a mean yield of 22.4 t/ha, 5.4 roots per plant, and $27.2 \%$ dry matter content. Kiroba is, however, is reported to be a high yielding variety with a potential production of $40.5 \mathrm{t} / \mathrm{ha}$ (Kundy et al., 2014). The low yields of Kiroba in our studies could be due to the lowly fertile sandy soils of NARI. Nyoka, Nanjenjeha, Musa Said, Mreteta, and Mbuyu were tolerant to CBSD and also similar to Kiroba in yield potential. These can be promoted directly for farmer cultivation in disease affected regions. Some other landraces such as Ntonto and Sumu ya Panya were susceptible to CBSD but had desirable root qualities including high yield potential. These can be used in cassava breeding programs.

The most effective and realistic way of reducing cassava losses due to CBSD is by deploying resistant and tolerant varieties. Cassava landraces identified to be resistant or tolerant in this study already have desirable root traits including high yields and dry matter content and preferred by farmers. These can be multiplied and used for direct cultivation or in breeding for minimizing the impact of CBSD on affected communities. Landraces with high yields, dry matter content and harvest index but susceptible to CBSD can be exploited for their superior agronomic characteristics. The Amani researchers of the 1940s and 1950s developed several disease resistant cassava varieties (Nichols, 1947; Jennings, 1957). Although this program has long closed with the departure of the British from eastern Africa, and an official collection of their material was not maintained, however, they appear to have survived as 
landraces with local farmers. These lines should be typed molecularly to eliminate duplications as well as to determine their pedigrees.

\section{Conclusion}

The findings from this study have shown that the landraces from Tanzania had different responses to CBSD. Landraces with minimal CBSD foliar and root symptoms were categorized as resistant and included Chimaje, Mfaransa and Supa B, while tolerant landraces developed foliar symptoms with minimal root necrosis and included Kikwada, Mbuyu, and Nyoka. Suceptible landraces had severe root necrosis regardless of the degree of foliar symptom severity, and they included Kigoma Red and Sumu ya Panya. Some of the resistant/tolerant landraces had high root weight and dry matter content, and could be used by farmers to reduce CBSD losses. Correlation analysis revealed that the presence of CBSD symptoms reduces the amount of usable roots, root weight, and dry matter content. Percent sum of squares revealed that response to CBSD and other roots traits tested is mostly genetically controlled. However, the expression of a few landraces was affected by environment as low rainfall and temperature coincided with increased severity and incidence. Resistant landraces identified in this study can be used in cassava breeding programs for direct cultivation as well as transferring resistance to farmer-preferred varieties.

\section{Acknowledgements}

This work was funded by the African Union Commission (AUC) under the grant number AURG/2/141. The AUC in turn received funds from the European Union Commission.

\section{References}

Abaca, A., Kawuki, R., Tukamuhabwa, P., Baguma, Y., Pariyo, A., Alicai, T., Omongo, C. A., Bua, A., 2012. Evaluation of local and elite cassava genotypes for resistance to cassava brown streak disease in Uganda. Agron. J. 11, 65-72. http://dx.doi.org/10.3923/ja.2012.65.72.

Adams, P., Abidrabo, P., Miano, D., Alicai, T., Kinyua, Z., Clarke, J., Macarthur, R., Weekes, R., Laurenson, L., Hany, U., Peters, D., Potts, M., Glover, R., Boonham, N., Smith, J., 2013. High throughput real-time RT-PCR assays for specific detection of cassava brown streak disease causal viruses, and their application to testing of planting material. Plant Pathol. 62(1), 233-242. http://dx.doi.org/10.1111/j.1365-3059.2012.02622.x.

Aigbe, S. O., Remison, S. U., 2010. The influence of root rot on dry matter partition of three cassava cultivars planted in different agro-ecological environments. Asian. J. Plant. Pathol. 4, 82-89. http://dx.doi.org/10.3923/ajppaj.2010.82.89. 
Alicai, T., Omongo, C. A., Maruthi, M. N., Hillocks, R. J., Baguma, Y., Kawuki, R., Bua, A., Otim-Nape, G. W., Colvin, J., 2007. Re-emergence of cassava brown streak disease in Uganda. Plant Dis. 91, 24-29. https://doi.org/10.1094/PD-91-0024.

Amuge, T., Berger, D. K., Katari, M. S., Myburg, A. A., Goldman, S. L., Ferguson, M. E., 2017. A time series transcriptome analysis of cassava (Manihot esculenta Crantz) varieties challenged with Ugandan cassava brown streak virus. Sci. Rep. 7: 9747.

DOI:10.1038/s41598-017-09617-z

Boakye, P. B., Kwadwo, O., Isaac, A., Parkes, E. Y., 2013. Genetic variability of three cassava traits across three locations in Ghana. Afr. J. Plant Sci. 7(7), 265-267. http://dx.doi.org/10.5897/AJPS12.058.

Bredeson, J. V., Lyons, J. B., Prochnik, S. E.,Wu, G. A., Ha, C. M., Edsinger-Gonzales, E., Grimwood, J., Schmutz, J., Rabbi, I. Y., Egesi, C., Nauluvula, P., Lebot, V., Ndunguru, J., Mkamilo, G., Bart, R. S., Setter, T. L., Gleadow, R. M., Kulakow, P., Ferguson, M. E., Rounsley, S., Rokhsa, D. S., 2016. Sequencing wild and cultivated cassava and related species reveals extensive interspecific hybridization and genetic diversity. Nature Biotechnol. 34(5), 562-570. https://doi.org/10.1038/nbt.3535.

Cooper, J. I., Jones, A. T., 1983. Responses of plants to viruses: proposals for the use of terms. Phytopathol. 73, 127-128.

Gondwe, F. M. T., Mahungu, N. M., Hillocks, R. J., Raya, M. D., Moyo, C. C., Soko, M. M., Chipungu, F. P., Benesi, I. R. M., 2002. Economic losses experienced by small-scale farmers in Malawi due to cassava brown streak virus disease. In: Legg, J. P., Hillocks, R. J., (Eds.), Cassava Brown Streak Virus Disease: Past, Present, and Future. Proceedings of an International Workshop, Mombasa, Kenya, October 27-30, 2002. Natural Resources International Limited, Aylesford, UK, pp. 28 - 36.

Hillocks, R. J., Jennings, D. L., 2003. Cassava brown streak disease: a review of present knowledge and research needs. Int. J. Pest Manage. 49, 225-234. http://dx.doi.org/10.1080/0967087031000101061.

Hillocks, R. J., Raya, M. D., Mtunda, K., Kiozia, H., 2001. Effects of brown streak virus disease on yield and quality of cassava in Tanzania, J. Plant. Pathol. 149, 389-394.

Hillocks, R. J., Raya, M. D., Thresh, J. M., 1999. Distribution and symptom expression of cassava brown streak virus disease in southern Tanzania. Afr. J. Root Tuber Crops. 3, 57-62.

Hillocks, R. J., Raya, M., Thresh, J. M., 1996. The association between root necrosis and above-ground symptoms of brown streak virus infection of cassava in Southern Tanzania. Int. J. Pest Manage. 42, 285-289.

Jeremiah, S. C., Legg, J. P., 2008. Cassava brown streak virus disease: farmers' perspectives on a new outbreak of this disease from the Lake zone of Tanzania. http://www.youtube.com/watch?v=nCJdws9CnUw. 
Jennings, D., 1994. Breeding for resistance to African cassava mosaic geminivirus in East Africa. Trop. Sci. 34, 110-122.

Jennings, D., 1957. Further studies in breeding cassava for virus resistance. E. Afr. Agr. Forestry. J. 22, 213-219. http://dx.doi.org/10.1080/03670074.1957.11665107.

Kamau, J., Melis, R., Laing, M., Derera, J., Shanahan, P., Ngugi, E. C. K., 2011. Farmers' participatory selection for early bulking cassava genotypes in semi-arid Eastern Kenya $\mathbf{J}$. Plant Breed. Crop Sci. Vol. 3(3), 44-52.

Kang, B. C., Yeam, I., Jahn, M. M., 2005. Genetics of plant virus resistance. Annu. Rev. Phytopathol. 43, 581-621. http://dx.doi.org/10.1146/annurev.phyto.43.011205.141140.

Kanju, E., Mkamilo, G., Mgoo, V., Ferguson, M., 2010. Statistical evidence linking the zigzag stem habit with tolerance to cassava brown streak disease. Roots 12, 4-6.

Kanju, E., Mtunda, K. J., Muhanna, M., Raya, M. D., Mahungu, N. M., 2003. Management of cassava brown streak virus disease in Tanzania. In: Legg, J. P., Hillocks, R. J., (Eds.), Cassava brown streak disease: past, present and future. Proceedings of an International Workshop, Mombasa, Kenya, 27-30 October 2002. Natural Resources International Limited, 66-9.

Kawano, K., 1987. Inherent and environmental factors related to cassava varietal selection. In: E. Hershey (Eds), Cassava Breeding. A multidisciplinary review, CIAT, Cali, Colombia, pp. 207-226.

Kulembeka, H. P., Ferguson, M., Herselman, L., Kanju, E., Mkamilo, G., Masumba, E., Fregene, M., Labuschagne, M. T., 2012. Diallele analysis of field resistance to brown streak disease in cassava (Manihot esculenta Crantz) landraces from Tanzania. Euphytica 187, 277 288. http://dx.doi.org/10.1007/s10681-012-0730-0.

Kundy, A. C., Mkamilo, G. S., Misangu, R. N., 2014. Assessment and selection of superior genotypes among elite cassava genotypes by farmers and scientists in Southern Tanzania. J. Nat. Sci. Res. 7, 17.

Legg, J., Somado, E. A., Barker, I., Beach, L., Ceballos, H., Cuellar, W., Elkhoury, W., Gerling, D., Helsen, J., Clair Hershey, C., Jarvis, A., Kulakow, P., Kumar, L., Lorenzen, J., Lynam, J., McMahon, M., Maruthi, G., Miano, D., Mtunda, K., Ntawuruhunga, P., Okogbenin, E., Pezo, P.,Terry, E., Thiele, G., Thresh, M., Wadsworth, J., Walsh, S., Winter, S., Tohme, J., Fauquet, C., 2014. A global alliance declaring war on cassava viruses in Africa. Food Sec. 6, 231-248. http://dx.doi.org/10.1007/s12571-014-0340-x.

Legg, J. P., Jeremiah, S. C., Obiero, H. M., Maruthi, M. N., Ndyetabula, I., Okao-Okuja, G., Bouwmeester, H., Bigirimana, S., Tata-Hangy, W., Gashaka, G., Mkamillo, G., Alicai, T., Lavar Kumar, P., 2011. Comparing the regional epidemiology of the cassava mosaic and cassava brown streak virus pandemics in Africa. Virus Res. 159(2), 161-170. http://dx.doi.org/10.1016/j.virusres.2011.04.018. 
Legg, J. P., Hillocks, R. J., (Eds) 2003. Cassava brown streak virus disease: past, present and future. In: Proceedings of an International Workshop, Mombasa, Kenya, October 27-30, 2002. Natural Resources International Limited, Aylesford, UK, pp. 100.

Mahungu, N. M., Bidiaka, M., Tata, H., Lukombo, S, N'luta, S., 2003. Cassava brown streaklike symptoms in Democratic Republic of Congo. Roots 8, 8-9.

Maruthi, M. N., Bouvaine, S., Tufan, H. A., Mohammed, I. U., Hillocks, R. J., 2014. Transcriptional response of virus-infected cassava and identification of putative sources of resistance for cassava brown streak disease. PLoS ONE 9(5), e96642.

https://doi.org/10.1371/journal.pone.0096642.

Maruthi, M. N., Hillocks, R. J., Mtunda, K., Raya, M. D., Muhanna, M., Kiozia, H., Rekha, A. R., Colvin, J., Thresh, J. M., 2005. Transmission of cassava brown streak virus by Bemisia tabaci. J. Phytopath. 153: 307-312.

Maruthi, M. N., Jeremiah, S. C., Mohammed, I. U., Legg, J. P., 2017. The role of the whitefly, Bemisia tabaci (Gennadius), and farmer practices in the spread of cassava brown streak ipomoviruses. J. Phytopathol. 00, 1-11. https://doi.org/10.1111/jph.12609

Masumba, E. A., Kapinga, F., Mkamilo, G., Salum, K., Kulembeka, H., Rounsley, S., Bredeson, J. V., Lyons, J. B., Rokhsar, D. S., Kanju, E., Katari, M. S., Myburg, A. A., van der Merwe, N. A., Ferguson, M. E., 2017. QTL associated with resistance to cassava brown streak and cassava mosaic diseases in a bi-parental cross of two Tanzanian farmer varieties, Namikonga and Albert. Theor. Appl. Genet. 1 - 22. https://doi.org/10.1007/s00122-017$2943-\mathrm{z}$

Mbazibwa, D. R., Tian, Y. P., Tugume, A. K., Mukasa, S. B., Tairo, F., Kyamanywa, S., Kullaya, A., Valkonen, J. P., 2009. Genetically distinct strains of cassava brown streak virus in the Lake Victoria basin and the Indian Ocean coastal area of East Africa. Arch. Virol. 154, 353-359. http://dx.doi.org/10.1007/s00705-008-0301-9.

Mohammed, I. U., Abarshi, M. M., Muli, B., Hillocks, R. J., Maruthi, M. N., 2012. The symptom and genetic diversity of cassava brown streak viruses infecting cassava in East Africa. Adv. Virol. 2012, 795697, 10 pages. http://dx.doi.org/10.1155/2012/795697.

Monger, W. A., Seal, S., Isaac, A. M., Foster, G. D., 2001. Molecular characterization of the cassava brown streak virus coat protein. Plant Pathol. 50, 527-534.

http://dx.doi.org/10.1046/j.1365-3059.2001.00589.x

Mugogo, S. E., Njapuka, A., 2007. Soil profile description and analytical interpretation at Chambezi and Naliendele in coastal Tanzania, In: G.S. Makamilo, E. Tenga (Eds.), Annual Research Report 2007/8, ARI Naliendele, Mtwara, Tanzania, 2007, pp. 56-67.

Mware, B., Narla, R., Amata, R., Olubayo, F., Songa, J., Kyamanywa, S., Ateka, E. M., 2009. Efficiency of cassava brown streak virus transmission by two whitefly species in coastal Kenya. J. Gen. Mol.Virol. 1, 40-45. http://www.academicjournals.org/jgmv. 
Ndunguru, J., Sseruwagi, P., Tairo, F., Stomeo, F., Maina, S., Djinkeng, A., Kehoe, M., Boykin, L. M., 2015. Analyses of twelve new whole genome sequences of cassava brown streak viruses and Ugandan cassava brown streak viruses from East Africa: diversity, supercomputing and evidence for further speciation. PLoS ONE. 10 (10), e0139321. https://doi.org/10.1371/journal.pone.0141939.

Nichols, R. F. J., 1950. The brown streak disease of cassava: distribution climatic effects and diagnostics symptoms. E. Afr. Agr. Forestry. J. 15, 154-160.

Nichols, R. F. W., 1947. Breeding cassava for virus resistance. E. Afr. Agr. Forestry. J. 12, 184-194.

Nzuki, I., Katari, M. S., Bredeson, J. V., Masumba, E., Kapinga, F., Salum, K., Mkamilo, G. S., Shah, T., Lyons, J. B., Rokhsar, D. S., Rounsley, S., Myburg, A. A., Ferguson, M. E., 2017. QTL mapping for pest and disease resistance in cassava and coincidence of some QTL with introgression regions derived from Manihot glaziovii. Front. Plant Sci. 8, 1168. https://doi.org/10.3389/fpls.2017.01168

Osogo, A. K., Muoma, J., Nyamwamu, P., Omuse, C. N., Were, H. K., 2014. Occurrence and distribution of cassava brown streak viruses in western Kenya. J. Agri-food Appl. Sci. 2(7), 184-190.

Pariyo, A., Baguma, Y., Alicai, T., Kawuki, R., Kanju, E., Bua, A., Omongo, C. A., Gibson, P., Osiro, D. S., Mpairwe, D., Tukamuhabwa, P., 2015. Stability of resistance to cassava brown streak disease in major agro-ecological zones of Uganda. J. Plant Breed. Crop Sci. 7, 66-78. http://dx.doi.org/10.5897/JPBCS2013.0490.

Pariyo, A., Tukamuhabwa, P., Baguma, Y., Kawuki, R. S., Alicai, T., Gibson, P., Kanju, E., Wanjala, B. W., Harvey, J., Nzuki, I., Rabbi, Y. I. and Ferguson, M. (2013). Simple sequence repeats (SSR) diversity of cassava in South, East and Central Africa in relation to resistance to cassava brown streak disease. Afr. J. Biotechnol 12, 4453-4464.

https://dx.doi.org/10.5897/AJB2013.12348

Patil, B., Legg, J. P., Kanju, E. E., Fauquet, C. M., 2014. Cassava brown streak disease: A threat to food security in Africa. J. Gen. Virol. 96(5), 956-968.

http://dx.doi.org/10.1099/vir.0.000014.

Rwegasira, G. M., 2009. Aspects of epidemiology of cassava brown streak virus disease in Tanzania. PhD Thesis. University of the Witwatersrand, South Africa, pp. 222.

SAS Institute Inc, 2013. SAS/STAT® 13.1 User’s Guide. SAS Institute, Cary, NC.

Tanzania Meteorological Agency (TMA), 2009. Precipitation, temperature and relative humidity data. United Republic of Tanzania.

Tumuhimbise, R., Melis, R., Shanahan, P., Kawuki, R., 2014. Genotype × environment interaction effects on early fresh storage root yield and related traits in cassava. Crop J. 2(5), 329-337. https://doi.org/10.1016/j.cj.2014.04.008. 
Vanderschuren, H., Moreno, I., Anjanappa, R. B., Zainuddin, I. M., Gruissem, W., Zhang, T., 2012. Exploiting the combination of natural and genetically engineered resistance to cassava mosaic and cassava brown streak viruses impacting cassava production in Africa. PLoS ONE. 7(9): e45277. https://doi.org/10.1371/journal.pone.0045277.

Winter, S., Koerbler, M., Stein, B., Pietruszka, A., Paape, M., Butgereitt, A., 2010. Analysis of cassava brown streak viruses reveals the presence of distinct virus species causing cassava brown streak disease in East Africa. J. Gen. Virol. 91(5), 1365-1372. http://dx.doi.org/10.1099/vir.0.014688-0. 